\title{
Os usuários de álcool, Atenção Primária à Saúde e o que é "perdido na tradução"
}

\author{
Bruno José Barcellos Fontanella ${ }^{1}$ \\ Marcelo Marcos Piva Demarzo ${ }^{2}$ \\ Guilherme Arantes Mello ${ }^{3}$ \\ Sandra Lúcia Correia Lima Fortes ${ }^{4}$
}

FONTANELLA, B.J.B. et al. Alcohol drinkers, Primary Health Care and what is "lost in translation". Interface - Comunic., Saude, Educ., v.15, n.37, p.573-85, abr./jun. 2011.

The paper discusses the paradox that is present in Primary Health Care (PHC): on the one hand, there are clinical technologies and opportunities to address patients who are problem drinkers and, on the other hand, it is verified that this kind of care is not effective for a considerable portion of those users. This paper is a critical and narrative bibliographic review whose results approach: the delimitation of this paradox; the health professionals' and their patients' difficulties to, respectively, provide and request interventions on patterns of problematic alcohol consumption; the applications and limitations of the motivational techniques, like the brief interventions in PHC; finally, some issues concerning professional training in health are discussed. It is concluded that it is necessary to promote a research agenda that deepens the understanding of the complex psychocultural meanings that this issue involves, particularly in Brazil.

Keywords: Primary health care. Alcoholrelated disorders. Clinical competence. Communication barriers.
Discute-se o paradoxo presente na Atenção Primária à Saúde (APS) de, por um lado, haver tecnologias clínicas e oportunidades para se abordarem pacientes que fazem uso problemático do álcool e, por outro, verificar-se que este tipo de atenção não se efetiva para parcela considerável desses usuários. Fez-se uma revisão bibliográfica crítica de cunho narrativo, cujos resultados abordam: a delimitação do paradoxo; as dificuldades pelas quais passariam os profissionais de saúde e seus pacientes para, respectivamente, disponibilizarem e solicitarem intervenções sobre padrões considerados problemáticos de consumo de álcool; as aplicações e limitações das técnicas motivacionais, como as intervenções breves, na APS, e, por fim, discutem-se algumas questões relativas à formação profissional em saúde. Conclui-se que esta problemática envolve a necessidade de se promover uma agenda de investigações que aprofunde a compreensão sobre seus complexos significados psicoculturais, particularmente no Brasil.

Palavras-chave: Atenção primária à saúde. Transtornos relacionados ao uso de álcool. Competência clínica. Barreiras de comunicação.

\footnotetext{
* Texto elaborado com base em apresentação oral no II Congresso

Paulista de Medicina de

Família e Comunidade (Fontanella, 2007). 1 Departamento de Medicina, Centro de Ciências Biológicas e da Saúde, Universidade Federal de São Carlos (UFSCar). Rodovia

Washington Luís, km 235. SP-310. São Carlos SP, Brasil. 13.565-905 bruno.fontanella@ gmail.com

${ }^{2}$ Departamento de Medicina Preventiva, Escola Paulista de Medicina, Universidade Federal de São Paulo. ${ }^{3}$ Departamento de Medicina, Centro de Ciências Biológicas e da Saúde, UFSCar. ${ }^{4}$ Departamento de Especialidades Médicas, Faculdade de Ciências Médicas, Universidade Estadual do Rio de Janeiro.
} 


\section{Introdução}

Este texto procura refletir sobre um paradoxo presente na Atenção Primária à Saúde (APS) e que envolve os profissionais clínicos e seus pacientes que fazem uso de bebidas alcoólicas. Trata-se da contradição de que uma atenção específica não se efetiva para uma parcela considerável desses usuários, a despeito das tecnologias clínicas e das oportunidades existentes para abordá-los.

Este paradoxo suscitou a metáfora, presente no título, sobre o que seria perdido na tradução nesta relação entre os clínicos e esses pacientes. Por que alguns saberes e práticas relativamente simples não são disponibilizados aos pacientes e, simetricamente, por que, muitas vezes, os desejos de mudança desses pacientes, em relação aos seus padrões de consumo de álcool, não se transformam em demandas?

Pressupõe-se haver obstáculos e ruídos tanto nos itinerários profissionais dos clínicos quanto nas trajetórias que levam pessoas a se tornarem pacientes (Zola, 1973). Este artigo discute este paradoxo, considerando, sobretudo, as supostas variáveis socioculturais envolvidas, que foram elencadas a partir do exame da literatura referente à APS.

\section{Método}

Trata-se de uma revisão crítica e narrativa, suscitada por inquietações advindas da experiência pedagógica e clínica dos autores, tanto em instituições de ensino médico quanto em instituições de saúde na APS.

Foi feito um levantamento bibliográfico junto ao Pubmed/Medline, Lilacs e Scielo, com a utilização de sintaxes que combinaram os seguintes termos: "álcool", "uso de risco", "abuso", "uso nocivo", "atenção primária à saúde", "atenção básica" e "intervenção breve", e seus equivalentes em inglês (para "atenção básica", somente em português). Dos 261 artigos resultantes, sobre variados aspectos da problemática, cerca de $20 \%$ foram julgados - a partir dos resumos - mais importantes para serem lidos e utilizados na discussão pretendida. Também foram consultados documentos oficiais de órgãos governamentais brasileiros e da Organização Mundial da Saúde (OMS).

Os pontos levantados para discussão foram agrupados em cinco sessões.

\section{Resultados e discussão}

\section{Delimitação do paradoxo}

Já em 1980, a OMS recomendava o desenvolvimento para a APS de estratégias de detecção precoce de usuários de álcool em risco de desenvolverem problemas (WHO, 1980). Seguiramse investigações que resultaram no AUDIT (Alcohol Use Disorder Identification Test), instrumento considerado, atualmente, eficaz e efetivo para suspeição diagnóstica dos diferentes padrões de consumo de álcool (Babor et al., 2001). Há hoje, também, a convicção de que a forma de abordagem dos profissionais da APS pode influenciar positivamente o padrão de ingestão de álcool de seus pacientes (Kaner et al., 2007; Conigliaro, Lofgren, Hanusa, 1998; Fleming et al., 1997) por meio das chamadas intervenções breves (IB) (Babor, Higgins-Biddle, 2001).

O recentemente publicado Regulamento do Sistema Único de Saúde (SUS) dedica 22 de seus setecentos e noventa artigos ao "Programa de Atenção Integral a Usuários de Álcool e outras Drogas" (Brasil, 2009), prevendo que a APS seja um dos principais componentes da atenção integral aos usuários de substâncias psicoativas. Trata-se, portanto, de uma reafirmação da agenda internacional de compromissos colocada, três décadas antes, aos pesquisadores, gestores e clínicos da Atenção Primária à Saúde.

O "Programa" preconiza que a APS se volte para a detecção precoce de casos de uso nocivo e de dependência de substâncias e para outras práticas preventivas, educativas e terapêuticas. A lógica da redução de danos e as técnicas de IB são mencionadas como forma de se alcançar o objetivo terapêutico 
de redução ou cessação do consumo, recomendando-se, também, o encaminhamento precoce de pacientes para intervenções mais específicas, incluindo a abordagem das complicações clínicas e/ou psiquiátricas.

Como contraponto à relevância e à disponibilidade desses recursos de abordagem, são conhecidas as dificuldades dos serviços e dos profissionais de saúde para diagnosticar, motivar, tratar ou encaminhar para tratamento os pacientes que fazem o chamado uso problemático de substâncias psicoativas.

A despeito dessas políticas indutoras e do desenvolvimento do AUDIT e das IB, muitos usuários de álcool adscritos aos serviços de APS continuam sem a oportunidade de ter seus comportamentos devidamente abordados (ressaltando-se que esta ênfase na APS não significa que dificuldades semelhantes não ocorram em outros cenários).

Quando se efetivam as atenções dos clínicos para o consumo de álcool de seus pacientes, dirigem-se, preferencialmente, para o uso nocivo ou para a síndrome de dependência, apesar de parte substancial dos problemas clínicos e de saúde pública ocorrer nos chamados bebedores de risco (hazardous drinkers) (McQuade et al., 2000).

As características de acesso irrestrito e preferencial da APS fazem com que as estimativas de prevalência, na população geral, de transtornos relacionados ao álcool devam ser cuidadosamente consideradas neste contexto. Enquanto a universalização dos atendimentos em APS não se efetivar, haverá uma discrepância entre os dados epidemiológicos da população geral e os dados da subpopulação efetivamente seguida. A priori, esperar-se-ia uma taxa maior de prevalência desta condição entre os frequentadores dos serviços, em razão da frequente co-ocorrência de agravos à saúde, lógica válida para qualquer condição clínica.

No entanto, as taxas de prevalência de dependência de álcool na APS parecem tender a ser menores, conforme será detalhado adiante, assim como parece ser também pequena a efetividade de ações voltadas para a suspeição diagnóstica. A título de exemplo, o SUS havia notificado, em 2004, no Sistema de Informação da Atenção Básica (SIAB), menos de 1\% deste tipo de condição em sua população cadastrada (Souza, 2005) - número pouco expressivo, mesmo considerando-se os vieses a que os dados dos sistemas de informação estão sujeitos (Silva, Laprega, 2005).

Parece provável que a vigilância aos problemas relacionados ao uso de álcool esteja, de fato, substancialmente abaixo do adequado neste cenário de atenção. Sendo assim em relação a um transtorno psiquiátrico clinicamente mais grave e chamativo (dependência), depreende-se que o reconhecimento dos demais padrões de uso de álcool (uso de risco e uso nocivo/abuso) seja ainda menos comum.

Embora possa ter contornos mais graves no Brasil, que há apenas duas décadas tem a APS como cenário estratégico e estruturante de um sistema sanitário universal e integral, o não-reconhecimento e a não-abordagem desses pacientes na APS são internacionalmente reconhecidos (Aalto et al., 2003; Aira et al., 2003; Kaner et al., 2001b; Arborelius, Damström Thakker, 1995; Rush et al., 1995).

Nos serviços em que uma demanda maior de pacientes é atendida e o tempo destinado a cada um é relativamente escasso, seria também útil conhecer e saber aplicar instrumentos de rastreamento (screening) para padrões problemáticos de uso de álcool, como o CAGE (Masur, Monteiro, 1983) e o AUDIT (Figlie et al., 2000), aumentando a eficiência da seleção de pacientes para intervenções motivacionais específicas. O rastreamento por meio desses instrumentos e o aconselhamento breve para uso problemático de álcool estão entre as intervenções mais custo-efetivas na APS, devendo, então, ser priorizadas (Solberg, Maciosek, Edwards, 2008).

\section{Os profissionais de saúde diante dos usuários de álcool}

A alta prevalência de transtornos mentais, de um modo geral, na população atendida na APS (Maragno et al., 2006), representaria, por si, um desafio para a atenção aos pacientes neste cenário (Tanaka, Lauridsen-Ribeiro, 2006). Os transtornos mentais promovem maiores dificuldades interpessoais entre pacientes e profissionais (Ballester et al., 2005), fenômeno comum e perceptível pelos próprios pacientes (Brêda, Augusto, 2001). 
No entanto, uma pesquisa brasileira com médicos trabalhando na função de generalistas na APS (com formações diversas) mostrou que eles tinham uma disposição apriorística para cuidar de pacientes com diagnósticos psiquiátricos, contanto que fossem capacitados para isso. Os participantes consideraram que seus pacientes teriam dificuldades para receber diagnósticos deste tipo e apontaram para a falta de recursos para encaminhá-los para uma atenção especializada na área (Ballester et al., 2005). Dentre os transtornos mentais, as dificuldades com o manejo de usuários de álcool e outras substâncias se sobressaem, podendo haver a percepção de inexistência de estratégias sistemáticas de manejo e de protocolos clínicos, consideradas inexistentes por profissionais pesquisados na década de 1990, quando ainda não eram tão difundidos os estudos com as intervenções breves (Rush et al., 1995).

Mais relevantemente, porém, do que essas dificuldades objetivas, os profissionais podem enfrentar barreiras subjetivas para abordar este tema durante uma consulta (Aira et al., 2003). Pode haver medo de prejudicar a relação médico-paciente caso o tema álcool seja levantado (Arborelius, Damström, Thakker, 1995), e alguns profissionais chegam a considerar essas questões como fora do âmbito da saúde (Roche, Guray, Saunders, 1991). O assunto pode ser considerado "sensível" e gerador de estigmas ao paciente, optando, alguns médicos, por não abordá-lo e por não registrar o diagnóstico nos prontuários (Aira et al., 2003). Haveria maior facilidade de se investigar o consumo quando se suspeita de problemas fisiopatológicos relacionados, o que representaria uma tendência a não abordar pessoas que fazem uso nocivo e de risco, mas sim, sobretudo, os dependentes. Alguns médicos também podem se ressentir da falta de ferramentas clínicas, como medicamentos para lidar com os estágios iniciais de problemas com o álcool.

A decisão final de investigar ou não o consumo pode depender de variáveis como idade, sexo e profissão do paciente, como, também, de sua aparência física (Aira et al., 2003). Algumas características dos usuários de álcool atendidos na APS de fato conferem-lhes maiores chances de serem alvos de IB: ser homem, estar desempregado e não ter educação universitária; mas, também, algumas características dos médicos se associam positivamente ao emprego desta técnica, como o tempo de treinamento e o tempo de duração de suas consultas (Kaner et al., 2001a).

Parte das dificuldades até aqui mencionadas parecem se relacionar, portanto, a uma postura de avaliação moral dos bebedores de risco; de outra forma, não seria compreensível considerar prejudicial a um paciente a abordagem de questões relacionadas a um problema de saúde ou a um comportamento de risco.

Outra questão de ordem cultural relaciona-se à categoria gênero, havendo a hipótese de que os comportamentos dos médicos e das médicas diferem quando estão lidando com usuários de álcool, sendo menor a probabilidade de as médicas realizarem IB (Aalto, Pekuri, Seppä, 2003).

Há também hipóteses consistentes sobre barreiras relacionadas às habilidades clínicas dos médicos. Se as IB são empregadas, as intervenções consideradas mais clínicas (escuta atenta e aconselhamento) seriam menos feitas do que as intervenções menos específicas (como a avaliação do consumo e a abordagem educativa) (Bradley et al., 2002). A chance de o tema ser abordado aumentaria quando o profissional é colocado em prontidão para agir, quando, por exemplo, seus pacientes foram previamente triados quanto ao padrão de consumo (Bradley et al., 2002).

A ocorrência de comorbidades psiquiátricas entre os usuários de álcool pode se relacionar a maiores dificuldades clínicas, podendo supor-se que cause ruídos adicionais durante os atendimentos.

\section{As dificuldades de os usuários de álcool chegarem aos serviços e demandarem ajuda}

Sinergicamente às dificuldades dos profissionais, muitas pessoas com problemas com uso de álcool não procuram ajuda formal (Kohn et al., 2005), embora possam fazê-lo por sintomas e condições relacionados (Fortes, Villano, Lopes, 2008).

Em determinados sistemas ou subsistemas de saúde, o consumo de álcool pode, inclusive, se relacionar negativamente com a utilização dos serviços de APS (Zarkin et al., 2004). Não procurando ou não conseguindo apoio no sistema formal de saúde, os pacientes talvez o busquem no sistema informal ou popular, ou promovam tentativas de autotratamento ou, mesmo, tenham seus quadros 
clínicos espontaneamente remitidos. Para esse subgrupo, o sistema formal não foi uma alternativa útil, pelo menos momentaneamente.

Várias barreiras impedem ou dificultam que uma pessoa que já reconheça intimamente ter uma relação problemática com alguma substância psicoativa recorra a serviços formais, não traduzindo seus desejos de diminuir, parar ou usar com menos riscos, na forma de demandas aos profissionais de saúde. Sabe-se que quanto mais problemático é o padrão de consumo, maior tende a ser a chance de um paciente querer discutir o assunto (Bradley et al., 2002).

Embora os homens tenham, em média, maiores chances de usar, de usar em maior quantidade e frequência e de serem dependentes de álcool, são eles, justamente, os que menos utilizam os serviços de saúde, inclusive da APS (Pinheiro et al., 2002).

Estudos epidemiológicos de 2001 e 2005 estimaram, respectivamente, em 11,2\% e 12,3\% a taxa de prevalência de dependência de álcool na população acima de 12 anos das cidades brasileiras com mais de duzentos mil habitantes (Carlini et al., 2006; Carlini et al., 2002). Nos dois levantamentos, os homens apresentaram taxas cerca de três vezes maiores que as mulheres, algo já ressaltado em estudos anteriores (Moreira et al., 1996; Almeida Filho et al., 1992; Rego et al., 1991).

A expectativa apriorística de que a taxa de prevalência fosse maior entre os frequentadores da APS não se confirma, ratificando a ideia de se tratar de um caminho para procura de tratamento caracteristicamente tortuoso e cheio de idas e vindas e desistências precoces (Thom, 1986), menos direto do que para problemas agudos e menos conflituosos. Disto parece resultar uma população flutuante nos serviços, que se refletiria em coeficientes de prevalência altamente variáveis.

O estudo de Villano (1998) detectou, entre homens atendidos em um ambulatório de medicina geral, $5,1 \%$ de uso nocivo e $12,4 \%$ de dependência $(N=91)$. Entre as mulheres, detectou-se 0,6 e $0,8 \%$, respectivamente $(N=393)$. Estudos mais recentes, em unidades do Programa de Saúde da Família, ratificam a ideia de que os problemas comunitários relacionados ao uso nocivo de álcool frequentemente não são cuidados nestas unidades, podendo chegar-se a taxas tão baixas quanto $1 \%$ dentre os pacientes portadores de transtornos mentais comuns, segundo avaliação do estado mental atual feita com o CIDI (Composite International Diagnostic Interview, para rastreamento de transtornos mentais) (Fortes, Villano, Lopes, 2008).

Em estudo com o AUDIT, realizado em unidades de APS (Magnabosco, Formigoni, Ronzani, 2007), detectou-se que $3,3 \%$ dos pacientes em atendimento (5,6\% em homens e $0,7 \%$ em mulheres) situavam-se na faixa de uso nocivo, justificando uma abordagem pelas equipes. Na faixa sugestiva de dependência e indicando um atendimento especializado, estavam 3,8\% (6,1\% em homens e 1,4\% em mulheres).

Estudo feito em unidades de atenção básica, também utilizando o AUDIT (Cordeiro et al., 2006), constatou que $34,37 \%$ dos homens e $2,29 \%$ das mulheres pesquisados foram considerados como fazendo uso nocivo de álcool. Noutro estudo (Vargas, Oliveira, Araújo, 2009), o mesmo instrumento revelou que 9,8\% dos pacientes de APS avaliados situavam-se na faixa IV (destes, 71,6\% eram homens e $22,4 \%$ mulheres).

\section{Aplicações e limitações das intervenções breves na Atenção Primária à Saúde}

Diante desses variados entraves envolvendo tanto os profissionais de saúde quanto seus potenciais pacientes, algumas iniciativas de divulgação e de pesquisa sobre as IB no Brasil já são registradas (Brasil, 2010; Corradi-Webster et al., 2005; Ronzani et al., 2005; Marques, Furtado, 2004). Elas se configuram como um conjunto sistematizado de intervenções que levam em consideração o padrão de uso e o momento vivido pelo paciente quanto à motivação para mudanças (Babor, HigginsBiddle, 2001); e, caracteristicamente, são voltadas para pacientes com envolvimentos com o álcool considerados menos graves. São relativamente simples e eficazes na redução da frequência do beber compulsivo, do beber excessivo, do consumo médio e do tempo de eventuais hospitalizações, sobretudo de homens (Kaner et al., 2007; Fleming et al., 1997), além de serem financeiramente eficientes para usuários e para os sistemas (Fleming et al., 2000). 
Instituindo as $I B$, os serviços deixam de se preocupar exclusivamente com os quadros mais extremos de dependência e do uso nocivo e, embora sem negar a gravidade psico e fisiopatológica desses quadros, deixam de enfatizar a prevenção secundária e terciária e passam a visar a prevenção primária e a promoção à saúde. Entretanto, para que sejam efetivas, os clínicos precisam estar aptos a realizá-las ao suspeitarem de uma situação de risco, o que também pode ser feito sistematicamente, e em larga escala, com o auxílio de instrumentos como o AUDIT.

Sabe-se que intervenções ou programas de educação continuada alteraram positivamente as habilidades dos profissionais de saúde em lidar com questões relativas ao álcool (Handmaker, Hester, Delaney, 1999), com boa relação custo-efetividade e interferindo positivamente na rotina assistencial e no prognóstico dos pacientes (Kaner et al., 2007; Conigliaro, Lofgren, Hanusa, 1998). A continuidade do tratamento e acompanhamento associa-se a melhores respostas e duração dos resultados (Minto et al., 2007) e, assim, a longitudinalidade, característica especifica da APS, aparece novamente como favorecedora do sucesso das IB.

Entretanto, determinados contextos locorregionais certamente demorarão a dispor de serviços de atenção secundária em saúde mental. Nesses casos, cabe à APS tratar, também, dos quadros de dependência e síndrome de abstinência alcoólica, seja ambulatorialmente, seja em internação domiciliar, e, inclusive, com a prescrição de psicofármacos específicos (O'Connor et al., 1997). Mas, mesmo nesses contextos, a prevenção primária e secundária por meio de técnicas como as IB são prioritárias, dada a frequência com que estariam indicadas.

Com relação à já referida eficácia das IB em reduzir o consumo de álcool, recente metanálise avaliou-a especificamente em contextos de clínica geral ou de APS (Kaner et al., 2007), confirmando a redução significativa do consumo de álcool entre os homens (hipótese já defendida antes, por exemplo, por Fleming et al., 1997), mas sem poder estatístico para concluir o mesmo entre as mulheres - o que sugere possíveis limitações da IB ou, pelo menos, a necessidade de mais estudos na subpopulação feminina, também conforme já hipotetizado antes (Anderson, 1993).

Uma limitação já claramente apontada das IB se refere ao modelo de aplicação, que deve ocorrer durante um atendimento realizado por profissional que mantenha com o paciente uma relação estável de confiança. Por isso, este modelo pode não se adequar a parcelas importantes da população que fazem uso de risco, mas que raramente mantêm um acompanhamento longitudinal de saúde, como é o caso dos adolescentes - para quem os formatos de rastreamento e IB precisariam ser adaptados (Yoast, Fleming, Balch, 2007), por exemplo, para a internet (Kypri, Saunders, Gallagher, 2003). Para o outro extremo etário, não foram localizados estudos de IB, a despeito de os problemas relacionados ao álcool serem frequentes na população idosa da APS (Lyness et al., 1999).

Um estudo em situações reais usando, em larga escala, o AUDIT, seguido de IB para quem obtivesse um escore positivo, mostrou uma efetividade abaixo da esperada e, para mulheres, resultados negativos. A hipótese levantada foi a de que uma abordagem feita de maneira indistinta, automática e independentemente do motivo de procura de assistência, pode exacerbar resistências psicológicas (Beich et al., 2007), o que vai, portanto, ao encontro da ideia de que as IB fazem mais sentido se aplicadas contextualmente em acompanhamentos longitudinais.

\section{Formação médica e de outras profissões da área da saúde}

Embora não se tenham detalhes sobre a formação dos profissionais participantes dos estudos até aqui mencionados (em particular, quanto se especializaram nas especificidades da APS), seus resultados têm implicações para todas as etapas da educação para profissionais de saúde.

Tradicionalmente, a abordagem de pacientes que fazem uso problemático de álcool tem sido feita fora da APS, por psiquiatras que, por este motivo, também não abordariam os quadros não patológicos (uso de risco). A psiquiatria, certamente, conta com recursos semiológicos de avaliação psicopatológica pouco aprendidos nos cursos de graduação e nas etapas educacionais posteriores, que são necessários para avaliar com destreza os sinais e sintomas que distinguem os diversos padrões de uso de álcool, porém exigem uma capacitação ainda não contemplada pelos conteúdos e métodos de aprendizagem dirigidos aos que não trabalharão na área da saúde mental (Hodges, Inch, Silver, 2001). 
Instrumentos padronizados podem diminuir este gap; sabe-se, porém, que a validade de aplicação de instrumentos padronizados fica limitada para algumas categorias de problemas mentais em razão de dificuldades semiológicas básicas dos aplicadores (Quintana et al., 2007). Em tese, o mesmo pode ocorrer com instrumentos como o CAGE e o AUDIT, próprios para o screening de problemas clínicos relacionados ao álcool.

São certamente necessárias habilidades cognitivas relacionadas às teorias que sustentam o modelo biopsicossocial, incluindo conhecimentos básicos de fisio e psicopatologia. À medida que as informações para os possíveis tipos de diagnósticos relacionados ao uso de álcool são colhidas exclusivamente durante a anamnese, junto ao paciente e seus familiares, prescindindo de exames laboratoriais, são requeridas, também, habilidades psicomotoras para uma adequada realização da entrevista e do exame psíquico.

Essas habilidades devem ser permeadas por outras da dimensão afetiva e ética, como: a primazia do juízo de conhecimento sobre o juízo de valor, o reconhecimento de preconceitos sociais e culturais, a compreensão das diferenças de poder na relação entre o médico e a pessoa em cuidado (e do potencial de abuso deste poder), o reconhecimento do medo de abordar o que é diferente ou o que pode comprometer os valores do próprio profissional (por exemplo, no caso, ser, ele próprio, um usuário de álcool, problemático ou não). Tais habilidades estão imbricadas na capacidade de se abordarem questões relacionadas à saúde de modo geral, sobretudo no contexto das grandes diferenças socioculturais entre profissionais e pacientes, como costuma ocorrer no SUS.

Refletindo sobre a formação em APS na realidade brasileira, a qualidade média dos serviços ainda é questionada, e um dos motivos disso é a incipiente capacitação profissional específica, a despeito da progressiva priorização da APS dentre as estratégias de reorganização do SUS, evidenciada pelo atual número de equipes de saúde da família, em torno de vinte e nove mil.

Esforços dos Ministérios da Saúde e da Educação (Brasil, 2005b) procuram valorizar os programas de residência em Medicina de Família e Comunidade (MFC) e criar programas de residência multiprofissional em Saúde da Família, ambos com foco em APS. No mesmo sentido, ações visando a especialização e a capacitação permanente dos profissionais em serviço culminaram recentemente com a integração de ações na forma de uma Universidade Aberta do SUS (UnA-SUS). Ademais, há, também, a valorização da APS na graduação em medicina e demais cursos da saúde via Diretrizes Curriculares Nacionais (DCN) e programas de indução, tais como o Pró-Saúde e Pet-Saúde (Brasil, 2007). Frente a esse positivo cenário institucional, pergunta-se: a formação específica em saúde mental é incentivada nessas iniciativas?

$\mathrm{Na}$ ausência de dados específicos que alicercem uma resposta, algumas inferências podem ser feitas a partir da análise dos editais e programas das ações mencionadas acima. Uma proposta que tende a valorizar a formação em saúde mental em APS é a propagação dos programas de residência multiprofissional em Saúde da Família. Esses programas incluem a psicologia como uma das profissões estratégicas (Brasil, 2005a), tendo, também, como perspectiva, a capacitação dessa categoria profissional para atuação junto aos Núcleos de Apoio à Saúde da Família (NASF) (Brasil, 2008), que têm a saúde mental como um eixo prioritário.

Em relação aos programas de residência em MFC, a valorização também é explícita nas portarias ministeriais e nos documentos da sociedade que agrega esses médicos especialistas, que abordam o conteúdo programático e de competência mínimo esperado, prevendo capacitação em saúde mental (Anderson et al., 2007; Brasil, 2006; Del Ciampo, Ricco, Daneluzzi, 2003).

Quanto às iniciativas de especialização em serviço, sua diversidade dificulta inferências, mesmo que superficiais. A UnA-SUS ainda não tem diretrizes de conteúdo definidas. Por sua vez, a Secretaria Nacional de Políticas sobre Drogas (SENAD) promove, há alguns anos, apoiada pela Divisão de Atenção Básica do Ministério da Saúde, um curso cuja meta é atingir milhares de profissionais de saúde e capacitá-los à distância para detecção e manejo de uso abusivo e dependência de substâncias psicoativas (Brasil, 2010). Em documento da Sociedade Brasileira de MFC sobre a especialização, a formação em saúde mental é explicitamente anunciada como prioritária (Castro Filho et al., 2007), devendo necessariamente fazer parte do currículo mínimo, com possível efeito indutor sobre os programas de especialização em APS. 
Já em relação aos cursos de graduação e às $D C N$, faz-se aqui um recorte específico quanto aos cursos de medicina (Brasil, 2001). Apesar do destaque para a promoção da saúde integral em todos os níveis do sistema, em nenhum momento há ênfase específica na formação para as ações de saúde mental, inclusive na configuração proposta para o internato médico. Mesmo estando a formação geral em saúde mental implícita em todo o documento, talvez fosse interessante explicitá-la, como se fez com outras grandes áreas do conhecimento médico.

Restaria, além disso, outra pergunta, também ainda sem resposta em território brasileiro: qual o impacto real dessas iniciativas de formação? Seriam custo-efetivas e suficientes para melhorar a qualidade da formação no que se refere à saúde mental?

\section{Comentários finais}

A presente revisão tem como limitação o desconhecimento das exatas formações dos profissionais que participaram dos estudos comentados. Certamente, nem todos foram capacitados nas especificidades clínicas encontradas neste cenário, o que é particularmente relevante de ser considerado no atual momento do processo de estruturação da APS brasileira.

No Brasil, há ainda dificuldades adicionais a serem enfrentadas em relação às encontradas em países europeus ou norte-americanos, que produziram parte dos estudos aqui comentados.

São escassos os estudos sobre as decorrências da diversidade brasileira de hábitos, costumes, valores e tradições no dia a dia dos serviços. O País conta, por exemplo, com heterogêneas subpopulações rurais que representam cerca de um quinto da população. No meio urbano, encontramse, também, diferentes subgrupos culturais, correspondendo a diferentes hábitos em relação ao álcool. Adicionalmente, a estratificação social brasileira sui generis acrescenta mais fatores de complexidade social, dificilmente captáveis em estudos epidemiológicos.

Considere-se, também, que as comentadas diferenças entre os gêneros requerem adaptações para tornar a APS mais atraente para a população feminina usuária de álcool (assim como para a população masculina geral), raciocínio extensível a outras configurações de gênero ainda menos estudadas, como os homossexuais e transexuais.

Especificidades locais tornam improvável que algumas ferramentas clínicas, como as IB, possam ser aplicadas em todos os territórios das unidades de saúde da APS, o que foi ilustrado na fala de um médico presente ao II Congresso Paulista de Medicina de Família e Comunidade (2007). Trabalhando numa Unidade de Saúde da Família cujo território compreende uma favela paulistana, avaliou ser impossível a aplicação das IB visando o consumo de álcool em seus pacientes, por considerar que os traficantes do local veriam isso como uma ameaça ao seu mercado.

Considere-se, ainda, que as ferramentas clínicas representadas pelos instrumentos de rastreamento, embora estudadas também "em nosso meio", foram validadas considerando, sobretudo, se não exclusivamente, a variável idioma. Ademais, atente-se para que os instrumentos padronizados de diagnóstico e abordagem de pacientes são acessórios da atividade clínica, sendo particularmente úteis quando se visa aumentar a eficiência de um serviço. A tradição clínica sugere, entretanto, que os profissionais de saúde procurem se adaptar aos seus pacientes, e não o contrário. Os diagnósticos de transtornos relacionados ao álcool são clínicos, e o principal instrumento de que a medicina dispõe neste caso é a história e o exame psíquico, prescindindo a priori da aplicação de questionários, testes, escalas e exames subsidiários.

Assim, a necessidade de eficiência e efetividade requer que o repertório profissional compreenda ambas as possibilidades de atuação (a protocolar e a clínica tradicional). Certamente, é importante saber aplicar um instrumento de screening, mas, para interpretar os resultados, é necessário compreender plenamente a problemática conceitual envolvida no espectro de fenômenos biológicos e socioculturais presentes no uso de álcool e nos diagnósticos relacionados.

Neste mesmo sentido, para aproximar o clínico de seus pacientes, seja na entrevista clínica "livre", seja durante a aplicação de instrumentos de rastreamento e intervenção, é desejável que o profissional de saúde, o médico em particular (enquanto formulador de diagnósticos nosológicos), se assegure de 
que entende o que seus pacientes dizem e, igualmente, de que seus pacientes entendem o que ele, o profissional, diz.

A etnografia chama este atributo de competência cultural (Bernard, 1988), ou seja, uma real competência quanto à compreensão do universo semântico, de valores, de tradições e apreensões dos pacientes. Na tradição da antropologia cultural, o pioneiro Malinowski já havia se referido à importância de "efetivamente" e "realmente" estar em contato com aquele que se quer conhecer (Malinowski, 1984, p.21-2). Transposto para a área da saúde, acreditamos que este tipo de competência tenderia a diminuir o que é "perdido na tradução", estando a serviço da competência clínica.

Contanto que se efetivem, as atuais diretrizes das políticas públicas brasileiras para educação profissional e para a atenção à saúde (integralidade do cuidado, acompanhamento longitudinal das pessoas e famílias, acesso universal, coordenação, advocacia pelo paciente), caso aplicadas às questões de saúde mental, em específico aos transtornos relacionados ao uso de álcool, suscitam uma perspectiva otimista.

Visando contribuir para esta efetivação, parecem imprescindíveis esforços investigativos que, progressivamente, detectem e permitam uma compreensão minuciosa do espectro de características e de dificuldades dos profissionais e de seus pacientes usuários de álcool. Advoga-se aqui, particularmente, em favor de uma agenda de investigações sobre os complexos significados psicoculturais que esta problemática envolve, como se pôde depreender da presente revisão.

\section{Colaboradores}

Bruno José Barcellos Fontanella responsabilizou-se pela concepção e redação do manuscrito original e pela organização das várias versões subsequentes, que resultaram na presente versão final. Marcelo Marcos Piva Demarzo, Guilherme Arantes Mello e Sandra Lúcia Correia Lima Fortes responsabilizaram-se por acréscimos críticos, conceituais e de redação durante a elaboração do manuscrito.

\section{Agradecimento}

Agradecemos à Profa. Dra. Ana Maria Galdini Raimundo Oda, pela leitura e comentários sobre o texto.

\section{Referências}

AALTO, M.; PEKURI, P.; SEPPÄ, K. Obstacles to carrying out brief intervention for heavy drinkers in primary health care: a focus group study. Drug Alcohol Rev., v.22, n.2, p.169-73, 2003.

AALTO, M. et al. The role of general practitioners' working style and brief alcohol intervention activity. Addiction, v.98, n.10, p.1447-51, 2003.

AIRA, M. et al. Factors influencing inquiry about patients' alcohol consumption by primary health care physicians: qualitative semi-structured interview study. Fam. Pract., v.20, n.3, p.270-5, 2003.

ALMEIDA FILHO, N. et al. Estudo multicêntrico de morbidade psiquiátrica em áreas urbanas brasileiras (Brasília, São Paulo, Porto Alegre). Rev. ABP APAL, v.14, n.3, p.93-104, 1992.

ANDERSON, M.I.P. et al. Bases para expansão e desenvolvimento adequados de programas de Residência em Medicina de Família e Comunidade. Rev. Bras. Med. Fam. Com., v.3, n.11, p.180-98, 2007.

ANDERSON, P. Effectiveness of general practice interventions for patients with harmful alcohol consumption. Br. J. Gen. Pract., v.43, n.374, p.386-9, 1993. 
ARBORELIUS, E.; DAMSTRÖM THAKKER, K. Why is it so difficult for general practitioners to discuss alcohol with patients? Fam. Pract., v.12, n.4, p.419-22, 1995.

BABOR, T.F.; HIGGINS-BIDDLE, J.C. Brief intervention for hazardous and harmful drinking: a manual for use in primary care. S/L.: Department of Mental Health and Substance Dependence, World Health Organization, 2001.

BABOR, T.F. et al. AUDIT - the alcohol use disorders identification test: guidelines for use in primary care. Geneva: Department of Mental Health and Substance Dependence, World Health Organization, 2001.

BALLESTER, D.A. et al. The general practitioner and mental health problems: challenges and strategies for medical education. Sao Paulo Med. J., v.123, n.2, p.72-6, 2005.

$\mathrm{BEICH}, \mathrm{A}$. et al. Screening and brief intervention targeting risky drinkers in Danish general practice: a pragmatic controlled trial. Alcohol Alcohol., v.42, n.6, p.593-603, 2007.

BERNARD, H.R. Research methods in cultural antropology. Newbury Park: Sage, 1988.

BRADLEY, K.A. et al. Alcohol-related discussions during general medicine appointments of male VA patients who screen positive for at-risk drinking. J. Gen. Intern. Med., v.17, n. 5, p.315-26, 2002.

BRASIL. Presidência da República. Secretaria Nacional de Políticas sobre Drogas. Curso SUPERA. Disponível em: <http://www.senad.gov.br/saiba_mais/capacitacao.html>. Acesso em: 7 abr. 2010.

. Ministério da Saúde. Portaria no 2.048/GM, de 03/09/2009. Aprova o Regulamento do Sistema Único de Saúde (SUS) e dá outras providências. Brasília: Ministério da Saúde, 2009. Disponível em: <http://bvsms.saude.gov.br/bvs/saudelegis/ gm/2009/prt2048 0309 2009.html>. Acesso em: 9 mar. 2011.

Ministério da Saúde. Portaria no 154, de 24 de janeiro de 2008. Cria os Núcleos de Apoio à Saúde da Família - NASF. Brasília: Ministério da Saúde, 2008. Disponível em: <http://189.28.128.100/dab/docs/legislacao/portaria154_04_03_08_re.pdf >. Acesso em: 9 mar. 2011.

. Ministério da Educação. Ministério da Saúde. Portaria Interministerial no 1507 , de 22 de junho de 2007. Institui o Programa de Educação pelo trabalho para a Saúde PET-Saúde. Brasília: Ministério da Educação/Ministério da Saúde, 2007. Disponível em: <http://dtr2001.saude.gov.br/sas/PORTARIAS/Port2007/GM/GM-1507.htm > . Acesso em: 9 mar. 2011.

. Ministério da Educação. Secretaria de Educação Superior. Comissão Nacional de Residência Médica. Resolução 02/2006, de 17 de maio de 2006. Dispõe sobre requisitos mínimos dos programas de residência médica e dá outras providências. Brasília: Ministério da Educação, 2006. Disponível em: <http://portal.mec.gov.br/dmdocuments/ resolucao02 2006.pdf>. Acesso em: 9 mar. 2011.

Ministério da Educação. Ministério da Saúde. Portaria Interministerial n 2117 , de 03 de novembro de 2005. Institui no âmbito do Ministério da Saúde e do Ministério da Educação a Residência Multiprofissional em Saúde. Brasília: Ministério da Educação, 2005a. Disponível em: <http://portal.saude.gov.br/portal/arquivos/pdf/2117.pdf>. Acesso em: 9 mar. 2011.

Ministério da Educação. Ministério da Saúde. Portaria Interministerial no $\overline{\mathbf{2 1 1 8}}$ de 03 de novembro de 2005. Institui parceria entre o Ministério da Educação e o Ministério da Saúde para cooperação técnica na formação e desenvolvimento de recursos humanos na área da saúde. Brasília: Ministério da Educação/Ministério da Saúde, 2005b. Disponível em: <http://portal.saude.gov.br/portal/arquivos/pdf/B\%202118\% 20 INSTITUIR\% 20PARCERIA \% 20ENTRE\% 200\% 20\% 20MEC\% 20e\% 200\% 20\% 20MS\% 20 REF.pdf > . Acesso em: 9 mar. 2011. 
BRASIL. Ministério da Educação. Conselho Nacional de Educação. Câmara de Educação Superior. Resolução CNE/CES n4, de 07 de novembro de 2001. Institui as Diretrizes Curriculares Nacionais do Curso de Graduação em Medicina. Brasília: Ministério da Educação, 2001. Disponível em: <http://portal.mec.gov.br/cne/arquivos/pdf/CES04. pdf>. Acesso em: 9 mar. 2011.

BRÊDA, M.Z.; AUGUSTO, L.G.D.S. O cuidado ao portador de transtorno psíquico na atenção básica de saúde. Cienc. Saude Colet., v.6, n.2, p.471-80, 2001.

CARLINI, E.A. et al. I Levantamento domiciliar sobre o uso de drogas psicotrópicas no Brasil: estudo envolvendo as 107 maiores cidades do país: 2001. São Paulo: Cebrid/ Unifesp, 2002.

II Levantamento domiciliar sobre o uso de drogas psicotrópicas no Brasil: estudo envolvendo as 108 maiores cidades do país: 2005. São Paulo: Cebrid/Unifesp, 2006.

CASTRO FILHO, E.D. et al. A especialização em medicina de família e comunidade e o desafio da qualificação médica para a Estratégia de Saúde da Família: proposta de especialização em larga escala via educação à distância. Rev. Bras. Med. Fam. Com., v.3, n.11, p.199-209, 2007.

CONIGLIARO, J.; LOFGREN, R.P.; HANUSA, B.H. Screening for problem drinking: impact on physician behavior and patient drinking habits. J. Gen. Intern. Med., v.13, n. 4, p.251-6, 1998.

CORDEIRO, Q. et al. Triagem para a identificação de uso nocivo de álcool na atenção primária à saúde. Rev. Assoc. Med. Bras., v.52, n.4, p. 200, 2006.

CORRADI-WEBSTER, C.M. et al. Capacitação de profissionais do Programa de Saúde da Família em estratégias de diagnóstico e intervenções breves para o uso problemático de álcool. SMAD, v.1, n.1, p.1-10, 2005. Disponível em: <http://pepsic.bvs-psi.org.br/ scielo.php?script=sci_arttext\&pid=\$1806-69762005000100004\&lng =pt\&nrm=iso $>$. Acesso em: 14 abr. $20 \overline{10}$.

DEL CIAMPO, L.A.; RICCO, R.G.; DANELUZZI, J.C. Residência médica em medicina geral e comunitária. Proposta de um programa de formação de médicos generalistas. Rev. Bras. Educ. Med., v.27, n.3, p.200-4, 2003.

FIGLIE, N.B. et al. The frequency of smoking and problem drinking among general hospital inpatients in Brazil - using the AUDIT and Fagerström questionnaires. Sao Paulo Med. J., v.118, n.5, p.139-43, 2000.

FLEMING, M.F. et al. Brief physician advice for problem alcohol drinkers. A randomized controlled trial in community-based primary care practices. JAMA, v.277, n.13, p.1039-45, 1997.

Benefit-cost analysis of brief physician advice with problem drinkers in primary care settings. Med. Care, v.38, n.1, p.7-18, 2000.

FONTANELLA, B.J.B. Encontros e desencontros: usuários de substâncias psicoativas e a Estratégia de Saúde da Família. In: CONGRESSO PAULISTA DE MEDICINA DE FAMíLIA E COMUNIDADE, 2., 2007, Campinas. Programa e Resumos ... Campinas: Associação Paulista de Medicina de Família e Comunidade, 2007. p.14.

FORTES, S.; VILLANO, L.A.B.; LOPES, C.S. Nosological profile and prevalence of common mental disorders of patients seen at the Family Health Program (FHP) units in Petrópolis, Rio de Janeiro. Rev. Bras. Psiquiatr., v.30, n.1, p.32-7, 2008.

HANDMAKER, N.S.; HESTER, R.K.; DELANEY, H.D. Videotaped training in alcohol counseling for obstetric care practitioners: a randomized controlled trial. Obstet. Gynecol., v.93, n.2, p.213-8, 1999.

HODGES, B.; INCH, C.; SILVER, I. Improving the psychiatric knowledge, skills, and attitudes of primary care physicians, 1950-2000: a review. Am. J. Psychiatry, v.158, n.10, p.1579-86, 2001. 
KANER, E.F. et al. Patient and practitioner characteristics predict brief alcohol intervention in primary care. Br. J. Gen. Pract., v.51, n.471, p.822-7, 2001a.

. Impact of alcohol education and training on general practitioners' diagnostic and management skills: findings from a World Health Organization collaborative study. J. Stud. Alcohol, v.62, n.5, p.621-7, 2001b.

KANER, E.F.S. et al. Effectiveness of brief alcohol interventions in primary care populations. Cochrane Database Syst. Rev., n.2, p.CD004148, 2007.

$\mathrm{KOHN}, \mathrm{R}$. et al. Los trastornos mentales en América Latina y el Caribe: asunto prioritario para la salud pública. Rev. Panam. Salud Publica, v.18, n.4-5, p.229-40, 2005. Disponível em: <http://www.scielosp.org/scielo.php?script=sci_arttext\&pid=\$1020-498920050009 $00002 \& \operatorname{lng}=e n \& n r m=i s o \& t \operatorname{lng}=e s>$. Acesso em: 7 abr. 2010.

KYPRI, K.; SAUNDERS, J.B.; CALLAGHER, S.J. Acceptability of various brief intervention approaches for hazardous drinking among university students. Alcohol Alcohol., v.38, n.6, p.626-8, 2003.

LYNESS, J.M. et al. Psychiatric disorders in older primary care patients. J. Gen. Intern. Med., v.14, n.4, p.249-54, 1999.

MAGNABOSCO, M.D.B.; FORMIGONI, M.L.O.D.S.; RONZANI, T.M. Avaliação dos padrões de uso de álcool em usuários de serviços de Atenção Primária à Saúde de Juiz de Fora e Rio Pomba (MG). Rev. Bras. Epidemiol., v.10, n.4, p.637-647, 2007.

MALINOWSKI, B. Argonautas do Pacífico ocidental: um relato do empreendimento e da aventura dos nativos nos arquipélagos da Nova Guiné Melanésia. 3.ed. São Paulo: Abril Cultural, 1984. (Coleção Os Pensadores).

MARAGNO, L. et al. Prevalência de transtornos mentais comuns em populações atendidas pelo Programa Saúde da Família (QUALIS) no Município de São Paulo, Brasil. Cad. Saude Publica, v.22, n.8, p.1639-48, 2006.

MARQUES, A.C.P.R.; FURTADO, E.F. Intervenções breves para problemas relacionados ao álcool. Rev. Bras. Psiquiatr., v.26, supl.1, p.28-32, 2004.

MASUR, J.; MONTEIRO, M.G. Validation of the "CAGE" alcoholism screening test in a Brazilian psychiatric inpatient hospital setting. Braz. J. Med. Biol. Res., v.16, n.3, p.215-8, 1983.

MCQUADE, W.H. et al. Detecting symptoms of alcohol abuse in primary care settings. Arch. Fam. Med., v.9, n.9, p.814-21, 2000.

MINTO, E.C. et al. Intervenções breves para o uso abusivo de álcool em atenção primária. Epidemiol. Serv. Saude, v.16, n.3, p.207-20, 2007.

MOREIRA, L.B. et al. Alcoholic beverage consumption and associated factors in Porto Alegre, a southern Brazilian city: a population-based survey. J. Stud. Alcohol, v.57, n.3, p.253-9, 1996.

O'CONNOR, P.G. et al. A preliminary investigation of the management of alcohol dependence with naltrexone by primary care providers. Am. J. Med., v.103, n.6, p.477-82, 1997.

PINHEIRO, R.S. et al. Gênero, morbidade, acesso e utilização de serviços de saúde no Brasil. Cien Saude Colet., v.7, n.4, p.687-707, 2002.

QUINTANA, M.I. et al. Validity and limitations of the Brazilian version of the Composite International Diagnostic Interview (CIDI 2.1). Rev. Bras. Psiquiatr., v.29, n.1, p.18-22, 2007.

REGO, R.A. et al. Epidemiologia do alcoolismo. Prevalência de positividade ao teste CAGE em inquérito domiciliar no Município de São Paulo. Rev. ABP- APAL, v.13, n.2, p.75-80, 1991.

ROCHE, A.M.; GURAY, C.; SAUNDERS, J.B. General practitioners' experiences of patients with drug and alcohol problems. Br. J. Addict., v.86, n.3, p.263-75, 1991.

RONZANI, T.M. et al. Implantação de rotinas de rastreamento do uso de risco de álcool e de uma intervenção breve na atenção primária à saúde: dificuldades a serem superadas.

Cad. Saude Publica, v.21, n.3, p.852-61, 2005. 
RUSH, B.R. et al. Early intervention for alcohol use: family physicians' motivations and perceived barriers. CMAJ, v.152, n.6, p.863-9, 1995.

SILVA, A.S.D.; LAPREGA, M.R. Avaliação crítica do Sistema de Informação da Atenção Básica (SIAB) e de sua implantação na região de Ribeirão Preto, São Paulo, Brasil. Cad. Saude Publica, v.21, n.6, p.1821-8, 2005.

SOLBERG, L.I.; MACIOSEK, M.V.; EDWARDS, N.M. Primary care intervention to reduce alcohol misuse ranking its health impact and cost effectiveness. Am. J. Prev. Med., v.34, n.2, p.143-52, 2008.

SOUZA, M.L.P.D. Expansão do Programa Saúde da Família (PSF) e identificação de problemas relacionados ao uso de álcool no Brasil. Rev. Bras. Psiquiatr., v.27, n.4, p.342-3, 2005.

TANAKA, O.Y.; LAURIDSEN-RIBEIRO, E. Desafio para a atenção básica: incorporação da assistência em saúde mental. Cad. Saude Publica, v.22, n.9, p.1845-53, 2006.

THOM, B. Sex differences in help-seeking for alcohol problems-1. The barriers to helpseeking. Br. J. Addict., v.81, n.6, p.777-88, 1986.

VARGAS, D.D.; OLIVEIRA, M.A.F.D.; ARAÚJO, E.C. Prevalência de dependência alcoólica em serviços de atenção primária à saúde de Bebedouro, São Paulo, Brasil. Cad. Saude Publica, v.25, n.8, p.1711-20, 2009.

VILLANO, L.A.B. Problemas psicológicos e morbidade psiquiátrica em serviços de saúde não psiquiátricos: o ambulatório de clínica geral. 1998. Tese (Doutorado) - Escola Paulista de Medicina, Universidade Federal de São Paulo, São Paulo. 1998.

WORLD HEALTH ORGANIZATION - WHO. Problems related to alcohol consumption. Geneva: Health Organization, 1980. (Technical Report Series, 650).

YOAST, R.A.; FLEMING, M.; BALCH, G.I. Reactions to a concept for physician intervention in adolescent alcohol use. J. Adolesc. Health, v.41, n.1, p.35-41, 2007.

ZARKIN, G.A. et al. Alcohol drinking patterns and health care utilization in a managed care organization. Health Serv. Res., v.39, n.3, p.553-70, 2004.

ZOLA, I.K. Pathways to the doctor-from person to patient. Soc. Sci. Med., v.7, n.9, p.677-89, 1973.

FONTANELLA, B.J.B. et al. Los usuarios de alcohol, la atención primaria de salud y lo que se "pierde en la traducción". Interface - Comunic., Saude, Educ., v.15, n.37, p.573-85, abr./jun. 2011.

El propósito es debatir la paradoja presentada en Atención Primaria de Salud (APS): existen tecnologías clínicas y oportunidades para hacer frente a los pacientes que hacen una utilización problemática del alcohol, y por outra parte, constatar que este tipo de atención no es eficaz para considerable parte de usuarios. Este artículo es una revisión bibliográfica crítica y narrativa que enfoca: la delimitación de esta paradoja, las dificultades de los profesionales de salud y de sus pacientes para a ofrecer y solicitar intervenciones en los patrones de consumo problemático; las aplicaciones y limitaciones de las técnicas de motivación, como las intervenciones breves, en APS y, por último, una discusión de algunas cuestiones relativas a la formación profesional en salud. Se concluye que es necesario promover una agenda de investigación que profundiza la comprensión de los significados psicoculturales complejos de esta cuestión, en particular en Brasil.

Palabras clave: Atención primaria de salud. Transtornos relacionados al uso del alcohol. Competencia clínica. Barreras de comunicación. 
